\title{
T4 Stage Finding
}

National Cancer Institute

\section{Source}

National Cancer Institute. T4 Stage Finding. NCI Thesaurus. Code C48732.

A clinical and/or pathologic primary tumor TNM finding indicating direct invasion of adjacent structures by cancer. 